\title{
Dietary choline is positively related to overall and cause-specific mortality: results from individuals of the National Health and Nutrition Examination Survey and pooling prospective data
}

\author{
Mohsen Mazidi $^{1 *}$, Niki Katsiki ${ }^{2}$, Dimitri P. Mikhailidis ${ }^{3}$ and Maciej Banach ${ }^{4,5,6}$ on behalf of the Lipid and \\ Blood Pressure Meta-Analysis Collaboration (LBPMC) Group \\ ${ }^{1}$ Key State Laboratory of Molecular Developmental Biology, Institute of Genetics and Developmental Biology, Chinese Academy \\ of Sciences, Chaoyang, Beijing, People's Republic of China \\ ${ }^{2}$ Second Propedeutic Department of Internal Medicine, Medical School, Aristotle University of Thessaloniki, Hippokration \\ Hospital, Thessaloniki, Greece \\ ${ }^{3}$ Department of Clinical Biochemistry, Royal Free Campus, University College London Medical School, University College London \\ (UCL), London, UK \\ ${ }^{4}$ Department of Hypertension, Chair of Nephrology and Hypertension, Medical University of Lodz, Lodz, Poland \\ ${ }^{5}$ Polish Mother's Memorial Hospital Research Institute (PMMHRI), Lodz, Poland \\ ${ }^{6}$ Cardiovascular Research Centre, University of Zielona Gora, Zielona Gora, Poland
}

(Submitted 26 March 2018 - Final revision received 28 November 2018 - Accepted 15 December 2018 - First published online 6 November 2019$)$

\section{Abstract}

Little is known about the association between dietary choline intake and mortality. We evaluated the link between choline consumption and overall as well as cause-specific mortality by using both individual data and pooling prospective studies by meta-analysis and systematic review. Furthermore, adjusted means of cardiometabolic risk factors across choline intake quartiles were calculated. Data from the National Health and Nutrition Examination Survey (1999-2010) were collected. Adjusted Cox regression was performed to determine the risk ratio (RR) and 95\% CI, as well as random-effects models and generic inverse variance methods to synthesise quantitative and pooling data, followed by a leave-one-out method for sensitivity analysis. After adjustments, we found that individuals consuming more choline had worse lipid profile and glucose homeostasis, but lower C-reactive protein levels $(P<0.001$ for all comparisons) with no significant differences in anthropometric parameters and blood pressure. Multivariable Cox regression models revealed that individuals in the highest quartile (Q4) of choline consumption had a greater risk of total (23\%), CVD (33\%) and stroke (30\%) mortality compared with the first quartile (Q1) $(P<0 \cdot 001$ for all comparison). These results were confirmed in a meta-analysis, showing that choline intake was positively and significantly associated with overall (RR $1 \cdot 12,95 \%$ CI $\left.1.08,1 \cdot 17, I^{2}=2.9\right)$ and CVD (RR 1.28, $95 \%$ CI 1.17, 1.39, $\left.I^{2}=9 \cdot 6\right)$ mortality risk. In contrast, the positive association between choline consumption and stroke mortality became non-significant (RR $1 \cdot 18,95 \%$ CI $0 \cdot 97,1 \cdot 43, P=0 \cdot 092, I^{2}=1 \cdot 1$ ). Our findings shed light on the potential adverse effects of choline intake on selected cardiometabolic risk factors and mortality risk.

Key words: Choline: Cardiovascular mortality: Overall mortality: Stroke mortality: Lipids: Inflammation: Glucose homeostasis

Choline is present in a wide variety of foods, with egg yolks and liver containing the largest amounts ${ }^{(1)}$. There is a considerable controversy regarding the beneficial/detrimental role of dietary choline intake. It has also been proposed that lower choline consumption may alter the epigenetic regulation for a series of genes which may accelerate the atherogenic process ${ }^{(2)}$. There is also important crosstalk between choline metabolism and the pathways of insulin sensitivity, fat deposition and energy metabolism through epigenetic modifications ${ }^{(3)}$. Furthermore, the metabolic pathway of dietary choline intake involving intestinal microbiota has been implicated in the pathogenesis of $\mathrm{CVD}^{(4)}$.

Some studies have shown that dietary choline consumption was inversely associated with C-reactive protein (CRP), interleukin-6 (IL-6) and tumour necrosis factor- $\alpha(\mathrm{TNF}-\alpha)^{(5,6)}$. Therefore,

Abbreviations: CRP, C-reactive protein; $\mathrm{HbA}_{1 \mathrm{c}}$, glycated $\mathrm{Hb}$; HR, hazard ratio; NHANES, National Health and Nutrition Examination Survey; RR, risk ratio; T2D, type 2 diabetes; TMAO, trimethylamine-N-oxide; WC, waist circumference. 
there is a hypothesis that choline intake may protect against CVD, by improving the inflammatory status ${ }^{(7)}$.

Evidence linking choline and insulin resistance (IR) is limited and largely based on animal experiments. Only one study in humans reported improved IR in individuals with a higher choline intake ${ }^{(8)}$. In another human study, serum choline levels were inversely associated with the risk for type 2 diabetes (T2D) ${ }^{(9)}$. Furthermore, decreased serum choline levels served as possible predictors of impaired glucose tolerance (IGT) and IR in the prediabetic state ${ }^{(10)}$. Just a single study examined the effects of dietary choline intake on body composition; a high dietary choline consumption was significantly associated with a favourable body composition ${ }^{(8)}$.

To the best of our knowledge, only two studies evaluated the link between CVD mortality and choline intake showing diverse results ${ }^{(11,12)}$, i.e. a Japanese study reported no association between intake of phosphatidylcholine and CVD mortality ${ }^{(12)}$, whereas an American study found a positive relationship between choline consumption and CVD mortality ${ }^{(11)}$. The Japanese study also evaluated the association between stroke mortality and choline intake, with null findings ${ }^{(12)}$. With regard to all-cause death, there is also just one study involving US adults, reporting a positive link with choline intake ${ }^{(11)}$. Furthermore, the Atherosclerosis Risk in Communities study (ARIC), involving 14430 middle-aged men and women, found a non-significant association between choline consumption and CVD incidence ${ }^{(13)}$. This finding was further supported by the Jackson Heart Study ( $n$ 3924), showing a positive relationship between choline intake and CHD incidence, but a reverse link between choline consumption and ischaemic stroke incidence ${ }^{(3)}$. In contrast, in another study in 16 165 women (age range: 49-70 years), there was no significant link between CVD and choline intake ${ }^{(14)}$. Further studies are needed to adjust for some confounders, such as fibre intake ${ }^{(5,15)}$, in order to elucidate the association between choline intake and CVD.

Given the paucity of studies and the controversial findings, we analysed a large and nationally representative sample of US adults to better understand the links of dietary choline with mortality and cardiometabolic risk factors. Furthermore, changes in cardiometabolic risk factors were cross-sectionally evaluated across choline intake quartiles. Finally, we systematically reviewed the literature for studies of dietary choline in relation to total and cause-specific mortality (CVD and stroke), and performed a random-effects meta-analysis to generate summary relative risks (RR).

\section{Methods \\ Original data}

Population. In the present study, results from both prospective and cross-sectional studies including data from the US National Health and Nutrition Examination Survey (NHANES) were used. The National Center of Health Statistics (NCHS) Research Ethics Review Board approved the underlying protocol and written informed consent was obtained from all participants. The current study is based on the analysis of data from two 2-year NHANES survey cycles between 1999 and 2010, restricted to participants aged $\geq 20$ years. Details on NHANES Laboratory/Medical
Technologists Procedures and Anthropometry Procedures are described elsewhere ${ }^{(16,17)}$.

Anthropometric data. A digital scale was used to measure weight to the nearest $100 \mathrm{~g}$ and a fixed stadiometer to measure height to the nearest $\mathrm{mm}$. BMI was calculated as weight in $\mathrm{kg}$ divided by the square of height in $\mathrm{m}$. Waist circumference (WC) was measured at the iliac crest to the nearest mm, using a steel tape ${ }^{(18)}$.

Biochemical parameters. A blood specimen was drawn from an antecubital vein. Glycated $\mathrm{Hb}\left(\mathrm{HbA}_{1 \mathrm{c}}\right)$ was measured using a Tosoh A1C 2.2 Plus Glycohemoglobin Analyzer (Tosoh Bioscience). Fasting blood glucose was measured by a hexokinase method using a Roche/Hitachi 911 Analyzer and Roche Modular P Chemistry Analyzer. Insulin was measured using an ELISA immunoassay (Mercodia) ${ }^{(19)}$. Other laboratory-test details are available in the NHANES Laboratory/Medical Technologists Procedures Manual ${ }^{(20)}$. Details on CRP measurement are available elsewhere ${ }^{(18)}$. The anthropometrically predicted visceral adipose tissue (apVAT) was calculated with sex-specific validated equations that included age, BMI, WC and thigh circumference $^{(21)}$. The equation for men was $6 \times$ WC $-4.41 \times$ proximal thigh circumference $+1.19 \times$ age -213.65 ; and the equation for women was $2.15 \times \mathrm{WC}-3.63 \times$ proximal thigh + $1.46 \times$ age $+6.22 \times$ BMI $-92.713^{(21)}$.

Dietary assessments. We have previously described the dietary assessments of NHANES ${ }^{(22)}$. Dietary intake was assessed via 24-h recall obtained by a trained interviewer, with the use of a computer-assisted dietary interview system with standardised probes, i.e. the United States Department of Agriculture (USDA) automated multiple-pass method (AMPM) ${ }^{(23,24)}$. Briefly, the type and quantity of all foods and beverages consumed in a single 24 -h period before the dietary interview (from midnight to midnight) were collected with the use of the AMPM. The AMPM is designed to enhance complete and accurate data collection while reducing respondent burden ${ }^{(24,25)}$. The USDA estimated the choline contents of NHANES foods in recipes by linking the ingredients in survey food recipes to food composition data provided by the USDA National Nutrient Database for Standard Reference. Total choline is the sum of free choline, glycerophosphocholine, phosphocholine, phosphatidylcholine and sphingomyelin.

Mortality. The anonymised data of NHANES 1999-2010 participants were linked to longitudinal Medicare and mortality data using the NHANES assigned sequence number. Mortality follow-up data are available from the date of the survey participation until 31 December 2011. We examined all-cause mortality, as well as mortality due to CVD (I00-I09, I11, I13, I20-I51, I60-I69) and cerebrovascular disease (I60-I69). Cause of death was determined using the 10th revision of the International Classification of Diseases (ICD-10).

Statistical analysis. The guidelines set by the Centers for Disease Control and Prevention ${ }^{(26)}$ were applied for analysis using a SPSS $^{\circledR}$ complex sample module version 22.0 (IBM Corp). 


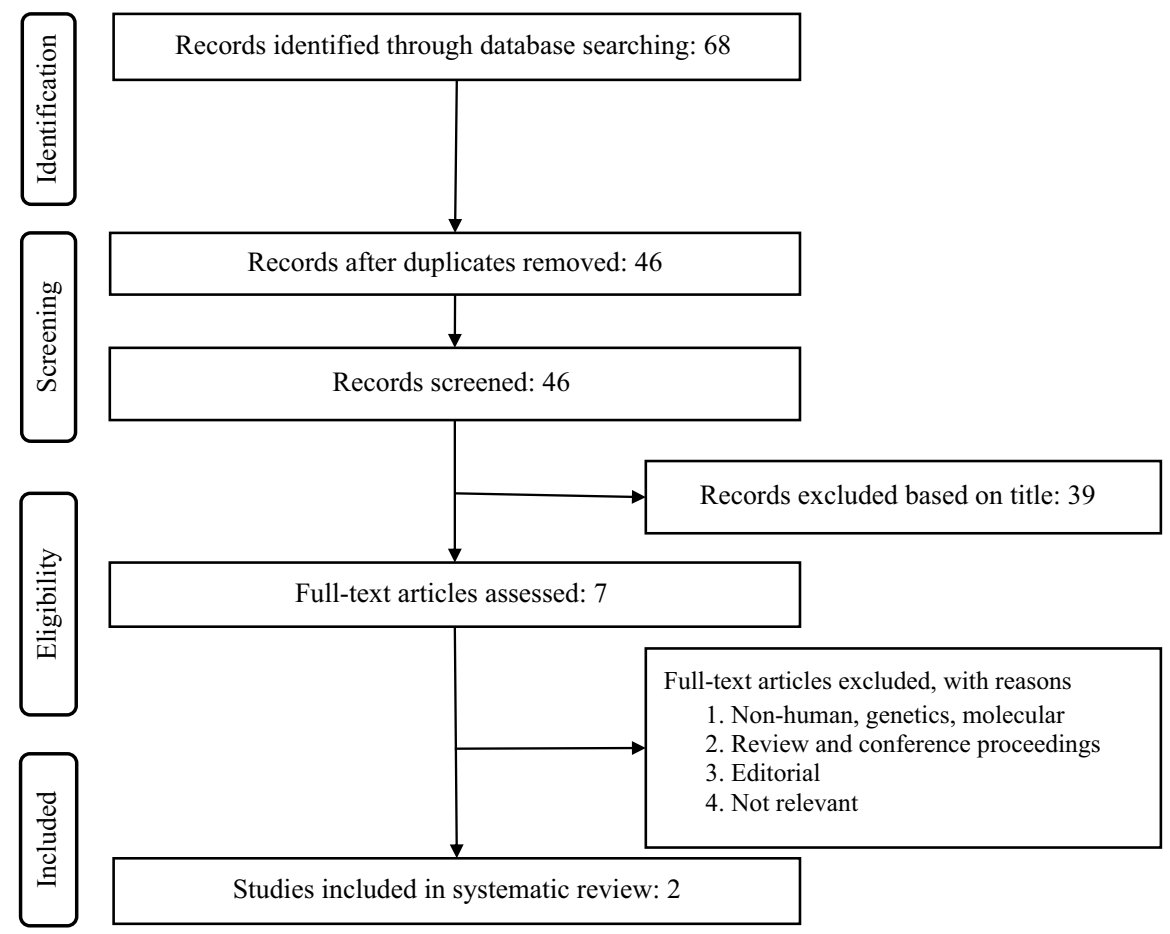

Fig. 1. Flow chart of the literature search for the meta-analysis on the relationship of choline consumption with overall and cause-specific mortality.

Continuous and categorical demographic variables were compared across the choline consumption quartiles using ANOVA and $\chi^{2}$ tests, respectively. Adjusted (for age, sex, race, education, marital status, poverty to income ratio, total energy intake, physical activity, smoking, alcohol consumption, intake of fibre and meat) means of cardiometabolic factors across choline consumption quartiles were calculated using ANCOVA. Adjusted (for age, sex, race, education, marital status, poverty to income ratio, total energy intake, physical activity, smoking, alcohol consumption, intake of fibre and meat) logistic regression was also performed to determine the risk of hypertension and T2D across choline intake quartiles; the results are expressed as OR and 95\% CI. The first quartile (Q1) was always used as reference for all models.

Multivariable Cox proportional hazards were applied to determine the hazard ratios (HR) and $95 \%$ CI of mortality (total, CVD and stroke) for choline consumption; the first quartile (Q1) was always used as reference for all models. To derive HR and $95 \%$ CI, two different models were used i.e. Model 1: Adjusted for age, sex, race, education, marital status, poverty to income ratio, total energy intake, physical activity, smoking, alcohol consumption, intake of fibre, polyunsaturated to saturated fat, saturated fat and folate; Model 2: Adjusted for age, sex, race, education, marital status, poverty to income ratio, total energy intake, physical activity, smoking, alcohol consumption, intake of fibre, PUFA, SFA, folate, BMI, hypertension and T2D. A two-sided $P<0.05$ was considered significant.

\section{Systematic review and meta-analysis}

Literature search and study selection. The present metaanalysis was designed, conducted and reported according to Meta-analysis of Observational Studies in Epidemiology
(MOOSE) guidelines ${ }^{(27)}$. The primary exposure of interest was choline consumption, while the primary outcome of interest was changes in total and cause-specific mortality subsequent to choline intake. Prospective cohort studies published up to January 2018 (without language restriction) were searched using PubMed, Embase, and Scopus databases; the query syntax of searching is shown in Table S1 in the online Supplementary material. This was complemented by hand searches of the reference list of eligible articles and email correspondence with authors for additional data, where relevant.

After excluding duplicates and based on titles and abstracts, we excluded studies on animals, or populations with baseline age $\geq 18$ years, prior $\mathrm{CHD}$, T2D or other chronic diseases. Eligible studies were selected by using predefined inclusion criteria of prospective cohort studies, healthy populations and original articles on the association of choline consumption and all-cause and cause-specific mortality (CHD and stroke). In addition, supplementary hand searching of reference lists of previous reviews or meta-analyses was conducted. Of eleven eligible full articles, two articles met the inclusion criteria (Fig. 1).

Study selection. Study selection started with the removal of duplicates, followed by screening of titles and abstracts by two reviewers (M. M. and M. B.). To avoid bias, they were blinded to the names, qualifications or institutional affiliations of the authors. The agreement between the reviewers was excellent ( $\kappa$ index: 0.91; $P<0 \cdot 001$ ). Disagreements were resolved at a meeting between reviewers prior to selected articles being retrieved (a flow chart is available in Fig. 1). We included studies if they met all the following criteria: (1) the point of interest was choline intake; (2) the studies were population-based cohort studies and reported cancer mortality data; (3) RR, HR or OR 
Table 1. Characteristics of the prospective cohort studies included in the meta-analysis

\begin{tabular}{|c|c|c|c|c|c|c|c|c|c|}
\hline $\begin{array}{l}\text { Author, year and } \\
\text { references }\end{array}$ & $\begin{array}{l}\text { Country, } \\
\text { region/cohort }\end{array}$ & $\begin{array}{l}\text { Men } \\
(\%)\end{array}$ & $\begin{array}{l}\text { Age } \\
\text { (years) }\end{array}$ & $\begin{array}{l}\text { Follow-up } \\
\text { duration } \\
\text { (years) }\end{array}$ & $\begin{array}{l}\text { No. of } \\
\text { cases } \\
\text { (mortality) }\end{array}$ & $\begin{array}{l}\text { No. of } \\
\text { subjects } \\
\text { (sample } \\
\text { size) }\end{array}$ & $\begin{array}{l}\text { Exposure } \\
\text { categories }\end{array}$ & Outcome & Main confounders \\
\hline Nagata et al..$^{(12)}$ & $\begin{array}{l}\text { Japan, The } \\
\text { Takayama } \\
\text { study }\end{array}$ & 45 & $>35$ & 16 & 982 & 29079 & $\begin{array}{l}\text { Choline } \\
\text { intake }\end{array}$ & CHD, stroke & $\begin{array}{l}\text { Age, energy, BMI, physical } \\
\text { activity, smoking status, } \\
\text { education, marital status, use } \\
\text { of vitamin supplements and } \\
\text { aspirin, history of diabetes } \\
\text { and hypertension, intake of } \\
\text { alcohol, SFA, PUFA:SFA, } \\
\text { folate, salt and fruits }\end{array}$ \\
\hline Zheng et al. ${ }^{(11)}$ & $\begin{array}{l}\text { USA, Nurses' } \\
\text { Health Study, } \\
\text { Health } \\
\text { Professionals } \\
\text { Follow-up } \\
\text { Study }\end{array}$ & 32 & $\begin{array}{c}46 \text { and } \\
52\end{array}$ & $\begin{array}{c}32 \text { and } \\
26\end{array}$ & 17829 & 120412 & $\begin{array}{l}\text { Choline } \\
\text { intake }\end{array}$ & $\begin{array}{l}\text { All-cause and } \\
\text { cardiovascular } \\
\text { mortality }\end{array}$ & $\begin{array}{l}\text { Age, BMI, white race, marital } \\
\text { status, menopausal status, } \\
\text { family history of CVD, smoking } \\
\text { status, alcohol consumption, } \\
\text { physical activity, history of } \\
\text { diabetes, hypertension, or } \\
\text { hypercholesterolaemia, aspirin } \\
\text { use, dietary intake of energy } \\
\text { and trans fat, ratio of poly- } \\
\text { unsaturated to saturated fat }\end{array}$ \\
\hline
\end{tabular}

estimates with $95 \%$ CI adjusted for multivariable factors were available or could be calculated. Studies were excluded according to the following criteria: (1) reviews, letters, unpublished results or comments; (2) those published in languages other than English; (3) not population-based cohort studies; (4) RR, HR or OR estimates with $95 \% \mathrm{CI}$ were not available or could not be calculated. Narrative reviews, comments, opinion papers, editorials, letters or any other publications lacking primary data and/ or explicit method descriptions, were also excluded.

Data extraction and management. The full text of studies meeting inclusion criteria was retrieved and screened to determine eligibility by two reviewers (M. M. and N. K.). The study quality assessment was performed according to the Newcastle-Ottawa Scale (Table S2 in Supplementary material) ${ }^{(28)}$. By evaluation of selection, comparability and outcome, the rating system scores studies from 0 (highest degree of bias) to 9 (lowest degree of bias). Additionally, we investigated the funding sources of all of the eligible studies. Following assessment of methodological quality, the two reviewers extracted data using a purpose-designed data extraction form and independently summarised what they considered to be the most important results from each study. These summaries were compared and any difference of opinion was resolved by discussion and consultation with a third reviewer (M. B.). Any further calculations on study data considered necessary were conducted by the first reviewer and checked by the second reviewer. Information extracted from each eligible study included the following items: author, year and references, country, study name, men (\%), age, follow-up time (years), no. of cases, no. of subjects, exposure categories, outcome and main confounders (Table 1).

Data synthesis and statistical analyses. For studies that reported results from different multivariable-adjusted models, the model with the majority of confounding factors was extracted from the meta-analysis. The random-effects model was applied to calculate pooled RR, $95 \% \mathrm{CI}$ and $P$ value for heterogeneity. RR comparing the highest with the lowest category were combined across studies to generate summary associations. The extent of heterogeneity across studies was examined using the $I^{2}$ test ${ }^{(29,30)} ; I^{2}>50 \%$ together with two-sided $P<0.05$ indicated significant heterogeneity ${ }^{(29,30)}$.

Publication bias. Potential publication bias was explored using visual inspection of Begg's funnel plot asymmetry, Begg's rank correlation and Egger's weighted regression tests. The Duval and Tweedie trim method was used to adjust the analysis for the effects of publication bias ${ }^{(31)}$. The present meta-analysis was conducted using the Comprehensive Meta-Analysis (CMA) V3 software (Biostat) ${ }^{(32)}$.

\section{Results \\ Original data}

Overall, 20325 individuals were included, with a mean age of 47.4 years and comprising $48.7 \%$ men and $51.3 \%$ women. Demographic characteristics of the participants are shown in Table 2. There was a significant age difference between quartiles of choline dietary intake; individuals in the highest quartile (Q4) were significantly younger than in the other quartiles $(P<0.001$, Table 2). Of note, from the lowest quartile (Q1) of choline dietary intake to the highest quartile (Q4), the percentage of men significantly increased (Q1: 29.4 v. Q4: 69.6\%), while the proportion of women decreased (Q1: 70.6 v. Q4: 39.4\%) (Table 2).

We also calculated adjusted (for age, sex, race, education, marital status, poverty to income ratio, total energy intake, physical activity, smoking, alcohol consumption, intake of fibre and meat) mean levels of cardiometabolic risk factors across increasing choline intake quartiles (Table 3). It was found that as 
Table 2. Characteristics of the study participants by choline consumption*

(Mean values with their standard errors; percentages)

\begin{tabular}{|c|c|c|c|c|c|c|}
\hline & & \multicolumn{4}{|c|}{ Choline consumption quartiles } & \multirow[b]{3}{*}{ Trend $P$} \\
\hline & & Q1 ( $n$ 5058) & Q2 ( $n$ 5070) & Q3 ( $n$ 5104) & Q4 ( $n$ 5093) & \\
\hline \multicolumn{2}{|c|}{ Dietary choline (mg), 50th (25th-75th)... } & $206(140-298)$ & $253(183-346)$ & $291(207-429)$ & $364(251-504)$ & \\
\hline \multirow[t]{2}{*}{ Age (years) } & Mean & $46 \cdot 6$ & $49 \cdot 1$ & $47 \cdot 2$ & $45 \cdot 1$ & $<0.001$ \\
\hline & $\mathrm{SE}$ & 0.3 & 0.3 & 0.2 & 0.2 & $<0.001$ \\
\hline \multirow[t]{2}{*}{ Sex } & Men (\%) & 29.4 & $39 \cdot 6$ & $52 \cdot 6$ & $69 \cdot 6$ & $<0.001$ \\
\hline & Women (\%) & $70 \cdot 6$ & $60 \cdot 4$ & $47 \cdot 4$ & 39.4 & \\
\hline \multirow{3}{*}{ Race/ethnicity } & Mexican-American (\%) & $17 \cdot 6$ & $17 \cdot 7$ & 19.5 & $21 \cdot 2$ & $<0.001$ \\
\hline & Non-Hispanic White (\%) & 43.4 & 48.5 & $48 \cdot 8$ & $47 \cdot 7$ & \\
\hline & Non-Hispanic Black (\%) & $24 \cdot 1$ & $20 \cdot 9$ & $19 \cdot 5$ & $19 \cdot 4$ & \\
\hline \multirow[t]{3}{*}{ Marital status } & Married (\%) & $46 \cdot 3$ & $50 \cdot 3$ & $55 \cdot 1$ & $53 \cdot 2$ & $<0.001$ \\
\hline & Widowed (\%) & 11.0 & $10 \cdot 1$ & $8 \cdot 1$ & 4.9 & \\
\hline & Divorced (\%) & $11 \cdot 0$ & 11.0 & $10 \cdot 6$ & $10 \cdot 1$ & \\
\hline \multirow[t]{3}{*}{ Education status } & Less than high school (\%) & $32 \cdot 9$ & $27 \cdot 9$ & $26 \cdot 9$ & $28 \cdot 1$ & $<0.001$ \\
\hline & Completed high school (\%) & 24.4 & 24.7 & $22 \cdot 7$ & $23 \cdot 8$ & \\
\hline & More than high school (\%) & 42.5 & $47 \cdot 3$ & $50 \cdot 3$ & $48 \cdot 1$ & \\
\hline
\end{tabular}

* Groups across the quartiles compared by either $\chi^{2}$ or ANOVA.

Table 3. Characteristics of the study participants by choline consumption adjusted for age, sex, race, education, marital status, poverty to income ratio, total energy intake, physical activity, smoking, alcohol consumption, intake of fibre and meat*

(Mean values with their standard errors)

\begin{tabular}{|c|c|c|c|c|c|c|c|c|c|}
\hline \multirow{4}{*}{ Dietary choline (mg), 50th (25th-75th)... } & \multicolumn{7}{|c|}{ Choline consumption quartiles } & & \multirow[b]{4}{*}{ Trend $P$} \\
\hline & \multirow{2}{*}{\multicolumn{2}{|c|}{$\begin{array}{l}\text { Q1 (n 5058) } \\
206(140-298)\end{array}$}} & \multirow{2}{*}{\multicolumn{2}{|c|}{$\begin{array}{l}\text { Q2 (n 5070) } \\
253(183-346)\end{array}$}} & \multirow{2}{*}{\multicolumn{2}{|c|}{$\begin{array}{l}\text { Q3 (n 5104) } \\
291(207-429)\end{array}$}} & \multirow{2}{*}{\multicolumn{2}{|c|}{$\begin{array}{l}\text { Q4 (n 5093) } \\
364(251-504)\end{array}$}} & \\
\hline & & & & & & & & & \\
\hline & Mean & SE & Mean & SE & Mean & SE & Mean & SE & \\
\hline BMI $\left(\mathrm{kg} / \mathrm{m}^{2}\right)$ & $27 \cdot 8$ & 0.1 & $28 \cdot 1$ & 0.1 & $27 \cdot 8$ & 0.1 & $27 \cdot 9$ & 0.1 & 0.392 \\
\hline Waist circumference $(\mathrm{cm})$ & $98 \cdot 2$ & 0.3 & $98 \cdot 1$ & 0.2 & $98 \cdot 6$ & 0.2 & $98 \cdot 8$ & 0.2 & 0.724 \\
\hline apVAT & $180 \cdot 2$ & 2.9 & $180 \cdot 7$ & $2 \cdot 2$ & $179 \cdot 2$ & $2 \cdot 2$ & $179 \cdot 3$ & 2.6 & 0.362 \\
\hline Systolic blood pressure (mmHg) & $123 \cdot 1$ & 0.1 & $120 \cdot 8$ & 0.2 & $121 \cdot 2$ & 0.3 & $122 \cdot 3$ & 0.2 & 0.215 \\
\hline Diastolic blood pressure $(\mathrm{mmHg})$ & 67.4 & 0.1 & $68 \cdot 2$ & 0.3 & 67.5 & 0.2 & $68 \cdot 1$ & 0.1 & 0.436 \\
\hline TAG (mg/dl)† & $154 \cdot 1$ & $2 \cdot 7$ & $155 \cdot 7$ & $2 \cdot 2$ & $155 \cdot 9$ & 2.9 & $157 \cdot 1$ & $2 \cdot 3$ & $<0.001$ \\
\hline HDL-cholesterol (mg/dl)† & $50 \cdot 6$ & 0.3 & $49 \cdot 9$ & 0.2 & $49 \cdot 6$ & 0.3 & $48 \cdot 6$ & 0.2 & $<0.001$ \\
\hline Fasting blood glucose (mg/dl)† & $98 \cdot 6$ & 0.5 & $99 \cdot 1$ & 0.6 & 99.9 & 0.7 & $100 \cdot 6$ & 0.4 & $<0.001$ \\
\hline Insulin $(\mu \mathrm{U} / \mathrm{ml})$ & $12 \cdot 9$ & 0.1 & $13 \cdot 1$ & 0.1 & $12 \cdot 8$ & 0.2 & $13 \cdot 0$ & 0.1 & 0.064 \\
\hline $\mathrm{HbA}_{1 \mathrm{c}}(\%)$ & 5.48 & 0.01 & 5.56 & 0.02 & $5 \cdot 68$ & 0.02 & $5 \cdot 72$ & 0.1 & 0.028 \\
\hline CRP $(\mathrm{mg} / \mathrm{dl}) \dagger$ & 0.45 & 0.01 & 0.40 & 0.01 & 0.38 & 0.01 & 0.35 & 0.02 & 0.026 \\
\hline
\end{tabular}

apVAT, anthropometrically predicted visceral adipose tissue; $\mathrm{HbA}_{1 \mathrm{c}}$, glycated $\mathrm{Hb}$; $\mathrm{CRP}, \mathrm{C}$-reactive protein.

${ }^{*}$ Adjusted means were compared across choline consumption by using ANCOVA.

† To convert TAG in $\mathrm{mg} / \mathrm{dl}$ to $\mathrm{mmol} / \mathrm{l}$, multiply by 0.0113 . To convert HDL in $\mathrm{mg} / \mathrm{dl}$ to $\mathrm{mmol} / \mathrm{l}$, multiply by 0.0259 . To convert glucose in $\mathrm{mg} / \mathrm{dl}$ to $\mathrm{mmol} / \mathrm{l}$, multiply by 0.0555 . To convert CRP in $\mathrm{mg} / \mathrm{dl}$ to $\mathrm{mg} / \mathrm{l}$, multiply by 10 .

individuals consumed more choline, they had a worse lipid profile (i.e. TAG and HDL-cholesterol) and glucose homeostasis (fasting blood glucose, $\mathrm{HbA}_{1 \mathrm{c}}$ ) but lower levels of inflammatory markers (CRP), while there was no difference in anthropometric parameters (BMI, WC and apVAT) and blood pressure (both systolic and diastolic, Table 3). For example, from the first (Q1) to the top quartile (Q4) of choline intake, CRP varied from 4.5 to $3.5 \mathrm{mg} / \mathrm{l}$, respectively ( $P=0.026$, Table 3 ), whereas fasting blood glucose and $\mathrm{HbA}_{1 \mathrm{c}}$ increased from $98.6 \mathrm{mg} / \mathrm{dl}(5.5 \mathrm{mmol} / \mathrm{l})$ and $5.48 \%$ to $100.6 \mathrm{mg} / \mathrm{dl}(5.6 \mathrm{mmol} / \mathrm{l})$ and $5.72 \%$, respectively ( $P<0.028$ for both comparisons, Table 3$)$.

By applying multiple logistic regressions adjusted for age, sex, race, education, marital status, poverty to income ratio, total energy intake, physical activity, smoking, BMI, CRP, alcohol consumption, intake of fibre and meat, we found that individuals with the highest choline intake (Q4) had a greater likelihood of T2D (OR 1·34, $95 \%$ CI 1·10, 1.99, P-trend < 0.001) compared with the first quartile (Q1), while no link was observed between the risk of hypertension and choline consumption $(P=0 \cdot 421)$.

During the follow-up period of 76.4 months, 3431 total deaths were recorded, including 967 CVD and 233 stroke deaths. The distribution of total and cause-specific mortality across choline intake quartiles is shown in Table 2.

Results from multivariable Cox regression models for risk of death (total, CVD and stroke) across choline intake quartiles are shown in Table 4. With regard to total mortality, in model 1, individuals in the highest (Q4) choline intake quartile had a $48 \%$ greater risk of total mortality (RR 1.48, $95 \%$ CI 1·06, 2.05, $P<0.001)$ compared with those in the first quartile (Q1); this association persisted even after further adjustment in model 2 (RR $1.23,95 \%$ CI $1.09,1.38, P<0.001$, Table 4 ). We found a 
Table 4. Sex-stratified multivariable-adjusted hazard ratios for mortality across choline consumption (Hazard ratios (HR) and $95 \%$ confidence intervals)

\begin{tabular}{|c|c|c|c|c|c|c|c|c|c|}
\hline \multirow{3}{*}{ Dietary choline $(\mathrm{mg})$, 50th (25th-75th)... } & & \multicolumn{7}{|c|}{ Choline consumption } & \multirow[b]{3}{*}{$P$-trend } \\
\hline & & \multirow{2}{*}{$\frac{206(140-298)}{H R}$} & \multicolumn{2}{|c|}{$253(183-346)$} & \multicolumn{2}{|c|}{ 291(207-429) } & \multicolumn{2}{|c|}{$364(251-504)$} & \\
\hline & & & $\mathrm{HR}$ & $95 \% \mathrm{Cl}$ & $\mathrm{HR}$ & $95 \% \mathrm{Cl}$ & $\mathrm{HR}$ & $95 \% \mathrm{Cl}$ & \\
\hline \multirow[t]{2}{*}{ Total mortality } & Model 1 & 1 (Reference) & 1.23 & $1.11,1.43$ & 1.28 & $1.14,1.43$ & 1.48 & $1.06,2.05$ & $<0.001$ \\
\hline & Model 2 & 1 (Reference) & 1.12 & $0.52,1.35$ & 1.17 & $1.09,1.25$ & 1.23 & $1.09,1.38$ & $<0.001$ \\
\hline \multirow[t]{2}{*}{ CVD mortality } & Model 1 & 1 (Reference) & 1.33 & $1 \cdot 19,1.48$ & 1.46 & $1.08,2.03$ & 1.78 & $1 \cdot 12,2 \cdot 82$ & $<0.001$ \\
\hline & Model 2 & 1 (Reference) & 1.22 & $0.97,1.54$ & 1.30 & $1.02,1.66$ & 1.33 & $1.19,1.48$ & $<0.001$ \\
\hline \multirow{2}{*}{ Cerebrovascular disease mortality } & Model 1 & 1 (Reference) & 1.49 & $0.88,2.50$ & 1.31 & $1.17,1.52$ & 1.42 & $1.27,1.96$ & $<0.001$ \\
\hline & Model 2 & 1 (Reference) & 1.27 & $0.86,1.88$ & $1 \cdot 15$ & $1.01,1.32$ & 1.30 & $1.02,1.66$ & $<0.001$ \\
\hline
\end{tabular}

${ }^{*}$ Model 1: adjusted for age, sex, race, education, marital status, poverty to income ratio, total energy intake, physical activity, smoking, alcohol consumption, intake of fibre, polyunsaturated to saturated fat and saturated fat.

† Model 2: adjusted for age, sex, race, education, marital status, poverty to income ratio, total energy intake, physical activity, smoking, alcohol consumption, intake of fibre, polyunsaturated to saturated fat, saturated fat, BMI, hypertension and diabetes.

positive and significant association between choline intake and CVD mortality, as individuals in the highest choline intake quartile had a $78 \%$ higher risk of CVD mortality compared with those in the first quartile; the same trend was found in model 2 (RR 1.33, $95 \%$ CI 1.19, 1.48, $P<0 \cdot 001$, Table 4). Dietary choline consumption was also positively and significantly associated with stroke mortality. For example, in model 2, individuals in the highest choline intake quartile had a $30 \%$ greater risk of stroke mortality compared with those in the first quartile (RR 1·30, $95 \%$ CI 1.02, 1·66, Table 4).

\section{Systematic review and meta-analysis}

Overviews of the characteristics of the two prospective cohort studies are shown in Table 1. A total of 149491 participants with 18811 mortality cases were included in the analysis. The duration of follow-up was $16^{(12)}$ and $32^{(11)}$ years, respectively, with a mean follow-up of 24.4 years. Results of quality assessment are shown in Table S2 in Supplementary material, with both studies scoring 9 .

Results of pooling data from prospective studies that evaluated the link between choline consumption, overall and cause-specific mortality are shown in Figs. 2-4. A positive and significant association was observed between choline intake and total mortality (RR $1 \cdot 12,95 \%$ CI 1.08, 1.17, $P<0 \cdot 001, n 3$ studies, Fig. 2), with no heterogeneity $\left(I^{2}=2 \cdot 9, P=0.882\right)$. Furthermore, choline intake predicted CVD mortality (RR $1 \cdot 28,95 \%$ CI $1 \cdot 17,1 \cdot 39, P<0.001$, $n 5$ studies, Fig. 3), i.e. individuals consuming more choline had a $28 \%$ greater risk of dying from CVD; no heterogeneity was observed $\left(I^{2}=9 \cdot 6, P=0 \cdot 864\right)$. However, the positive association between stroke mortality and choline consumption became non-significant (RR $1 \cdot 18,95 \%$ CI 0.97, 1.43, $P=0 \cdot 092, n 3$ studies, Fig. $\left.4, I^{2}=1 \cdot 1, P=0 \cdot 934\right)$.

Sensitivity analysis. In leave-one-out sensitivity analyses, the pooled effect estimates remained similar for the associations of choline consumption with total (RR 1.12, $95 \%$ CI 1.08, 1.17, $P<0.001)$, CVD $(1 \cdot 28,95 \%$ CI 1.17, 1.39, $P<0.001)$ and stroke mortality (RR 1.18, $95 \%$ CI $0.97,1.43, P=0.092)$. This confirms that the significant differences between the studied groups were the overall effect of all included studies.

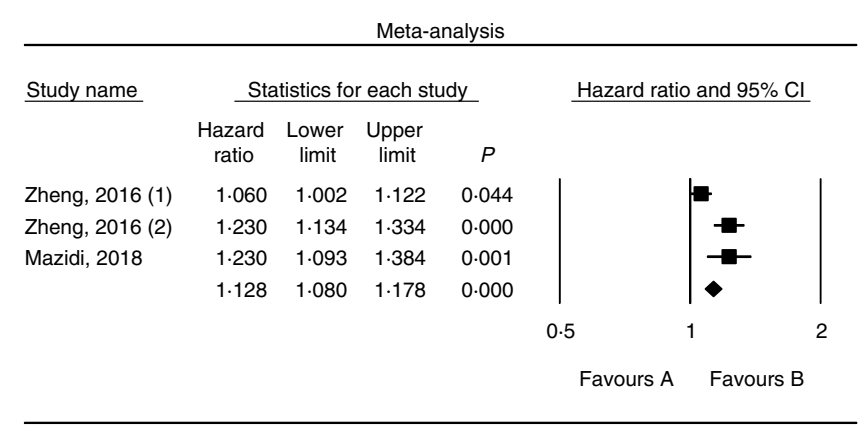

Fig. 2. Forest plot of choline consumption and risk of total mortality.

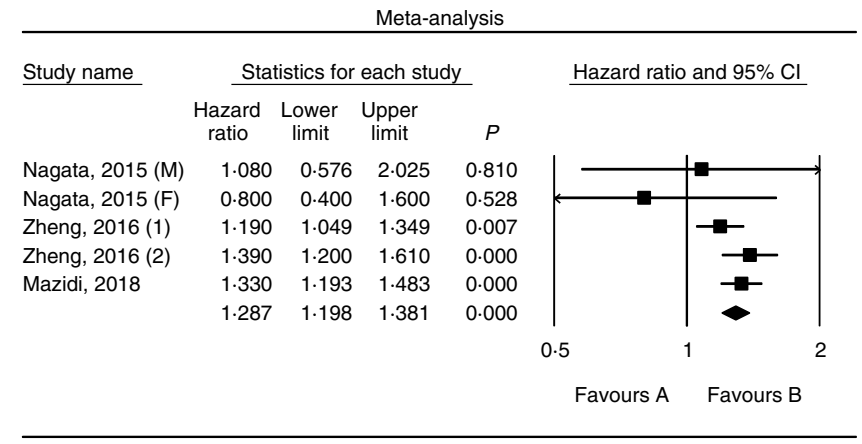

Fig. 3. Forest plot of choline consumption and risk of CVD mortality. M, male; $\mathrm{F}$, female.

Publication bias. Visual inspection of the funnel plot symmetry suggested no potential publication bias for the comparison of choline consumption and total mortality (Fig. S1 in Supplementary material). Furthermore, both Egger's linear regression (intercept $-1 \cdot 10,95 \%$ CI $-4 \cdot 02,1 \cdot 81$, two-tailed $P=0.314$ ) and Begg's rank correlation test (Kendall's tau with continuity correction $=-1 \cdot 01, \mathrm{z}=0 \cdot 244$, two-tailed $P=0 \cdot 806$ ) indicated no publication bias. After adjustment of the effect size for potential publication bias using the 'trim and fill' correction, no potentially missing studies were imputed in the funnel plot (Fig. S2 in Supplementary material). Furthermore, the 'fail-safe $\mathrm{N}$ ' test showed that thirty-two studies would be needed to bring 


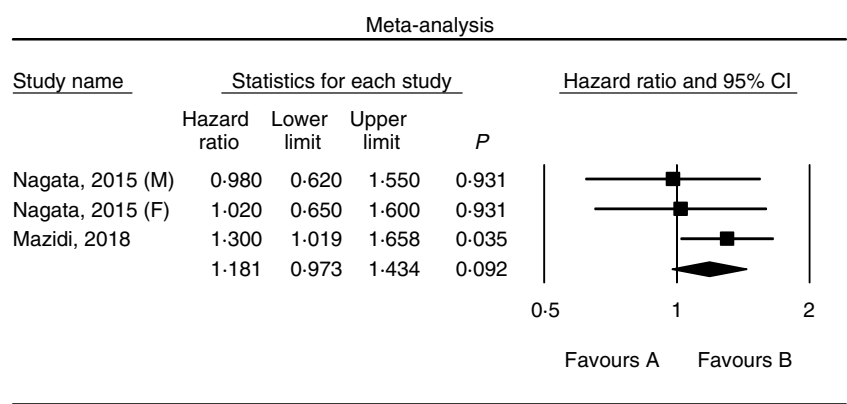

Fig. 4. Forest plot of choline consumption and risk of stroke mortality. M, male; $\mathrm{F}$, female.

the weighted mean difference down to a non-significant $(P>0.05)$ value.

\section{Discussion}

The present study evaluated the associations of dietary choline intake with overall and cause-specific mortality in a large, randomly selected, nationally representative US population. A positive link between total, CVD and stroke mortality with choline intake was observed, a finding that was further confirmed by conducting a meta-analysis. Furthermore, higher choline intake was associated with worse cardiometabolic risk factors, as well as a greater risk of T2D.

To the best of our knowledge, this is the first time that a positive link between choline intake and stroke mortality in US adults is reported. In contrast, a study performed among Japanese adults (13 355 males and 15724 females, aged >35 years, 16 years follow-up) found no significant association between choline consumption and stroke mortality ${ }^{(12)}$. Furthermore, in the present study, dietary choline intake was positively associated with total and CVD mortality. Until now, only a single study evaluated the link between all-cause mortality and choline intake; the authors examined the association between habitual dietary intake of phosphatidylcholine (which is the major dietary source of choline) with all-cause mortality among 80978 women and 39434 men followed for up to 32 years. They concluded that a higher intake of phosphatidylcholine was associated with an increased all-cause mortality (RR 1.11, $95 \%$ CI 1.06, 1.17) ${ }^{(11)}$. Another two studies evaluated the link between dietary choline intake and CVD mortality, one of them in a Japanese population with null findings (RR 0.80, 95\% CI $0.40,1.60)$ and another one in US adults with positive and significant results (RR 1.26, $95 \%$ CI 1.15, 1.39) ${ }^{(11,12)}$.

A few studies ( $n$ 4) evaluated the relationship between CVD events and choline consumption. For example, Dalmeijer et al. (Netherlands, $n 130667)^{(14)}$ and Millard et al. (USA, $\left.n 35316\right)^{(3)}$ reported no significant associations between choline intake and CVD incidence (RR 1.04, $95 \%$ CI 0.75, 1.45 and RR 0.97, $95 \%$ CI $0 \cdot 86,1 \cdot 09$, respectively) ${ }^{(3,14)}$. The same studies ${ }^{(14)}$ found no link between choline intake and stroke incidence (RR 0.86, $95 \% \mathrm{CI}$ $0.51,1.44$ and RR 0.94, 95\% CI 0.80, 1.11, respectively) ${ }^{(3,14)}$. Overall, several studies reported inconsistent associations between dietary choline intake and risk of $\mathrm{CHD}^{(6,13,14)}$.
The pathophysiological links between choline intake and mortality are not clear. Phospholipid phosphatidylcholine is a major dietary source of choline and trimethylamine, which are metabolised into circulating trimethylamine-N-oxide (TMAO) via intestinal microbiota-dependent mechanisms ${ }^{(4)}$. Higher circulating TMAO concentrations have been related to an increased risk of CVD and mortality ${ }^{(4)}$. Wang et al. found that plasma concentrations of metabolites (including TMAO) from dietary phosphatidylcholine via a gut microbiota-related nutrient metabolism pathway were highly predictive of CVD risk $^{(4)}$. Our study extends these findings by suggesting that the dietary sources of such gut microbiota-related metabolites may also be related to mortality. We found that the highest quartile (Q4) of choline consumption had a higher HR for overall, CVD and stroke mortality. Taken together our study (showing a significant and positive relationship between choline intake and CVD mortality) and other studies that evaluated the link between choline consumption and CVD incidence (reporting attenuated or non-significant associations) ${ }^{(6,13,14)}$, we could suggest that the effects of dietary choline intake may be greater on CVD prognosis than on CVD development.

It seems that high choline intake would increase expression and activity of betaine-homocysteine methyltransferase enzyme (BHMT) in the liver and kidney; this enzyme, by transferring a methyl group, would convert homocysteine to methionine and would lead to reduction in circulating homocysteine levels ${ }^{(33)}$. Choline is also a betaine precursor which may explain why high choline intake is associated with reduced homocysteine concentrations ${ }^{(34)}$. The TAG-raising effect of phosphatidylcholine may contribute to the positive association with the risk of $\mathrm{CVD}^{(35)}$. Furthermore, intestinal microbial metabolism of dietary phosphatidylcholine has been implicated in the pathogenesis of atherosclerosis $^{(4)}$.

The present study has several limitations. Although our analysis was adjusted for multiple factors, including sociodemographic characteristics, lifestyle habits, physical activities and energy intake, genetic and dietary nutrient factors absorbed when consuming choline-rich foods and unknown or poorly measured factors could not be completely ruled out. Furthermore, we need to mention that due to our observational design, even we adjusted for known confounders, there is still a chance of residual bias. This limitation might be addressed in the future by randomised control trials. It would also be interesting to have studies based on the source of choline intake. However, our findings may have public health implications, addressing the extent to which associations between TMAO, cardiometabolic risk factors and mortality may influence dietary advice related to the consumption of the TMAO precursor, i.e. choline.

In conclusion, we found that individuals consuming more choline had worse lipid profile and glucose homeostasis, but lower CRP levels and no significant differences in anthropometric parameters and blood pressure, compared with those consuming less choline. Furthermore, individuals in the highest quartile (Q4) of choline intake had a greater overall, CVD and stroke mortality than those in the lowest quartile. Our findings revealed that a higher choline intake could have detrimental effects on health but further research is needed in this field. 


\section{Acknowledgements}

There are no acknowledgements to be made.

The material presented in this manuscript is original and has not been submitted for publication elsewhere.

N. K. has given talks, attended conferences and participated in trials sponsored by Amgen, Angelini, Astra Zeneca, Boehringer Ingelheim, MSD, Novartis, NovoNordisk, Sanofi and WinMedica. D. P. M. has given talks and attended conferences sponsored by MSD, AstraZeneca and Libytec. The other authors have no conflict of interest to declare.

\section{Supplementary material}

For supplementary material/s referred to in this article, please visit https://doi.org/10.1017/S0007114519001065

\section{References}

1. Niculescu MD \& Zeisel SH (2002) Diet, methyl donors and DNA methylation: interactions between dietary folate, methionine and choline. J Nutr 132, 2333s-2335s

2. Zaina S, Lindholm MW \& Lund G (2005) Nutrition and aberrant DNA methylation patterns in atherosclerosis: more than just hyperhomocysteinemia? J Nutr 135, 5-8.

3. Millard HR, Musani SK, Dibaba DT, et al. (2018) Dietary choline and betaine; associations with subclinical markers of cardiovascular disease risk and incidence of CVD, coronary heart disease and stroke: the Jackson Heart Study. Eur J Nutr 57, 51-60.

4. Wang Z, Klipfell E, Bennett BJ, et al. (2011) Gut flora metabolism of phosphatidylcholine promotes cardiovascular disease. Nature 472, 57-63.

5. Rajaie S \& Esmaillzadeh A (2011) Dietary choline and betaine intakes and risk of cardiovascular diseases: review of epidemiological evidence. ARYA Atherosclerosis 7, 78-86.

6. Detopoulou P, Panagiotakos DB, Antonopoulou S, et al. (2008) Dietary choline and betaine intakes in relation to concentrations of inflammatory markers in healthy adults: the ATTICA study. Am J Clin Nutr 87, 424-430.

7. Mazidi M, Rezaie P, Kengne AP, et al. (2016) Gut microbiome and metabolic syndrome. Diabetes Metab Syndr 10, S150-S157.

8. Gao X, Wang Y, Randell E, et al. (2016) Higher dietary choline and betaine intakes are associated with better body composition in the adult population of Newfoundland, Canada. PLOS ONE 11, e0155403.

9. Chen L, Chen YM, Wang LJ, et al. (2013) Higher homocysteine and lower betaine increase the risk of microangiopathy in patients with diabetes mellitus carrying the GG genotype of PEMT G774C. Diabetes/Metab Res Rev 29, 607-617.

10. Li X, Chen Y, Liu J, et al. (2012) Serum metabolic variables associated with impaired glucose tolerance induced by high-fathigh-cholesterol diet in Macaca mulatta. Exp Biol Med (Maywood, NJ) 237, 1310-1321.

11. Zheng Y, Li Y, Rimm EB, et al. (2016) Dietary phosphatidylcholine and risk of all-cause and cardiovascular-specific mortality among US women and men. Am J Clin Nutr 104, 173-180.

12. Nagata C, Wada K, Tamura T, et al. (2015) Choline and betaine intakes are not associated with cardiovascular disease mortality risk in Japanese men and women. J Nutr 145, 1787-1792.

13. Bidulescu A, Chambless LE, Siega-Riz AM, et al. (2007) Usual choline and betaine dietary intake and incident coronary heart disease: the Atherosclerosis Risk in Communities (ARIC) study. BMC Cardiovasc Disord 7, 20.
14. Dalmeijer GW, Olthof MR, Verhoef P, et al. (2008) Prospective study on dietary intakes of folate, betaine, and choline and cardiovascular disease risk in women. Eur J Clin Nutr 62 , 386-394.

15. Meyer KA \& Shea JW (2017) Dietary choline and betaine and risk of CVD: a systematic review and meta-analysis of prospective studies. Nutrients 9 , E711.

16. Remer T (2001) Influence of nutrition on acid-base balance metabolic aspects. Eur J Nutr 40, 214-220.

17. Engberink MF, Bakker SJ, Brink EJ, et al. (2012) Dietary acid load and risk of hypertension: the Rotterdam study. Am J Clin Nutr 95, 1438-1444.

18. Centers for Disease Control and Prevention (2011) Laboratory Procedure Manual: C-Reactive Protein. http://www.cdc.gov/ NCHS/data/nhanes/nhanes_09_10/CRP_F_met.pdf (accessed August 2019).

19. Mazidi M, Michos ED \& Banach M (2017) The association of telomere length and serum 25-hydroxyvitamin D levels in US adults: the National Health and Nutrition Examination Survey. Arch Med Sci 13, 61-65.

20. Centers for Disease Control and Prevention (2019) National Center for Health Statistics, National Health and Nutrition Examination Survey. http://www.cdc.gov/nchs/nhanes.htm (accessed August 2019).

21. Samouda H, Dutour A, Chaumoitre K, et al. (2013) $\mathrm{VAT}=\mathrm{TAAT}-\mathrm{SAAT}$ : innovative anthropometric model to predict visceral adipose tissue without resort to CT-Scan or DXA. Obesity (Silver Spring) 21, E41-E50.

22. Mazidi M, Kengne AP, Mikhailidis DP, et al. (2018) Effects of selected dietary constituents on high-sensitivity C-reactive protein levels in U.S. adults. Ann Med 50,1-6.

23. Ahluwalia N, Andreeva VA, Kesse-Guyot E, et al. (2013) Dietary patterns, inflammation and the metabolic syndrome. Diabetes Metab 39, 99-110.

24. Ahluwalia N, Dwyer J, Terry A, et al. (2016) Update on NHANES dietary data: focus on collection, release, analytical considerations, and uses to inform public policy. Adv Nutr 7, 121-134.

25. Moshfegh AJ, Rhodes DG, Baer DJ, et al. (2008) The US Department of Agriculture automated multiple-pass method reduces bias in the collection of energy intakes. Am J Clin Nutr 88, 324-332.

26. Centers for Disease Control and Prevention (2015) Analytic and Reporting Guidelines. http://www.cdc.gov/nchs/data/nhanes /nhanes0304/nhanesanalyticguidelinesdec2005.pdf (accessed August 2019).

27. Stroup DF, Berlin JA, Morton SC, et al. (2000) Meta-analysis of observational studies in epidemiology: a proposal for reporting. Meta-analysis Of Observational Studies in Epidemiology (MOOSE) group. JAMA 283, 2008-2012.

28. Stang A (2010) Critical evaluation of the Newcastle-Ottawa scale for the assessment of the quality of nonrandomized studies in meta-analyses. Eur J Epidemiol 25, 603-605.

29. Mazidi M, Rezaie P, Gao HK, et al. (2017) Effect of sodium-glucose cotransport- 2 inhibitors on blood pressure in people with type 2 diabetes mellitus: a systematic review and meta-analysis of 43 randomized control trials with 22528 patients. J Am Heart Assoc 6, e004007.

30. Mazidi M, Kengne AP \& Banach M (2018) Effects of coenzyme Q10 supplementation on plasma C-reactive protein concentrations: a systematic review and meta-analysis of randomized controlled trials. Pharmacol Res 128, 130-136.

31. Duval S \& Tweedie R (2000) Trim and fill: a simple funnel-plotbased method of testing and adjusting for publication bias in meta-analysis. Biometrics 56, 455-463.

32. Borenstein M, Hedges L, Higgins J, et al. (2005) Comprehensive Metaanalysis (Vers. 2). Englewood Cliffs, NJ: Biostat Inc. 
33. Hajer GR, van der Graaf Y, Olijhoek JK, et al. (2007) Levels of homocysteine are increased in metabolic syndrome patients but are not associated with an increased cardiovascular risk, in contrast to patients without the metabolic syndrome. Heart 93, 216-220.

34. Schwab U, Torronen A, Toppinen L, et al. (2002) Betaine supplementation decreases plasma homocysteine concentrations but does not affect body weight, body composition, or resting energy expenditure in human subjects. Am J Clin Nutr 76, 961-967.

35. Olthof MR, van Vliet T, Verhoef P, et al. (2005) Effect of homocysteine-lowering nutrients on blood lipids: results from four randomised, placebo-controlled studies in healthy humans. PLoS Med 2, e135. 